\title{
Comparaison de l'efficacité de quelques procédés de décontamination surfacique
}

\author{
A. DUIGOU ${ }^{1}$, M. FATTAHI $^{1}$, J. FAZILEABASSE ${ }^{2}$
}

(Manuscrit reçu le 31 mars 2009, accepté le 9 juin 2009)

RÉSUMÉ L'étude de la décontamination surfacique des colis de déchets radioactifs est un projet important pour EDF, dans le cadre du démantèlement. Cette étude a deux objectifs : tout d'abord de réduire la dosimétrie due au contrôle de la noncontamination surfacique des colis de déchets radioactifs et ensuite garantir la non contamination surfacique des colis jusqu'à sa destination finale (site de traitement et/ou entreposage) en évitant la mise en suspension et le dépôt d'activité labile lors du transport des colis qui pourrait entraîner localement le dépassement du seuil de contamination surfacique réglementaire. Pour cela, une étude bibliographique des méthodes de décontamination proposées dans la littérature a été effectuée. Cette étude a permis de voir si des techniques pouvaient répondre aux critères choisis. Les techniques susceptibles de répondre aux exigences ont été testées afin d'évaluer leur efficacité. L'étude bibliographique et les essais de qualification ont montré qu'une méthode semblait plus adaptée que les autres à notre problématique.

ABSTRACT Comparison of the efficiency of several methods of surface decontamination.

The study of surface decontamination of radioactive waste packages is an important project for EDF in the context of decommissioning. This study has two objectives: firstly, to reduce dosimetry due to the control of non-contaminated area of radioactive waste packages and secondly to ensure the non-contamination of surface parcels as they are sent to their final destination (treatment site and/or storage). This serves to avoid the suspension and the filling of labile activity in the transport of packages that could lead to locally exceeding the regulatory surface contamination threshold. For that purpose, a bibliographical study of the methods of decontaminations proposed in the literature was made. This study allowed seeing if techniques could answer the chosen criteria. Techniques seeming to be able to answer the requirements were then tested to estimate their efficiency. The bibliographical study and the tries of qualification showed that a method seemed more adapted than the others to our problem.

Keywords: Dosimetry / nuclear power industry / decontamination / package

\section{Introduction}

La France est au premier rang mondial des producteurs d'énergie d'origine nucléaire : environ $80 \%$ de son électricité produite vient de réacteurs nucléaires.

1 SUBATECH, École des Mines de Nantes, 4 rue Alfred Kastler, BP 20722, 44307 Nantes Cedex 3, France.

2 EDF R\&D, Département Simulation et Traitement de l'information pour l'exploitation des Systèmes de production, 6 quai Watier, BP 49, 78401 Chatou Cedex, France. 
C'est Électricité de France (EDF), qui exploite ces 58 réacteurs nucléaires, répartis en 19 centrales. Cette production d'énergie s'accompagne d'une production de déchets radioactifs. Cette production conduit à prendre des mesures pour réduire la dosimétrie occasionnée par les contrôles réglementaires des transports des colis de déchets avant leur expédition vers les centres de traitement et/ou de stockage. En effet ce transport est soumis à une réglementation internationale, ordonnée par l'Agence internationale de l'énergie atomique, AIEA, qui limite la contamination surfacique labile à $4 \mathrm{~Bq} / \mathrm{cm}^{2}$. Afin de respecter cette réglementation, EDF soumet les colis à des contrôles systématiques pour assurer une contamination surfacique au moins dix fois moins importante que le seuil autorisé. Le retrait systématique de la contamination surfacique labile des colis de déchets pourrait assurer le respect des limites réglementaires d'exposition et garantir la non-agglomération de contamination engendrée par le transport et qui éventuellement pourrait entraîner un dépassement du seuil réglementaire. Elle permettrait également de réduire la dosimétrie, du personnel, engendrée par les contrôles.

Toutefois, la décontamination doit respecter un certain nombre de paramètres. Le critère le plus important est de travailler à sec, c'est-à-dire sans effluent liquide. Le retraitement des déchets liquides est un processus long et fastidieux qui créerait un problème plus important que la décontamination des colis. Un autre point important est la conservation de la nature et des propriétés de la surface des colis. Ces colis, après décontamination, sont destinés à être transportés vers des centres de traitement et/ou stockage. Ils ne doivent pas subir de modifications de surface (fissure...) sinon il pourrait y avoir des risques de fuite ce qui pose un problème important pour la sécurité et l'environnement. Enfin, le processus de décontamination doit être rapide, efficace, et applicable à l'échelle industrielle. Le processus devra donc respecter tous ces paramètres.

Les différentes méthodes de décontamination proposées dans la littérature ont été étudiées (laser, ultrasons...) afin de déterminer la mieux adaptée à notre problématique qui est d'enlever la contamination labile faisant l'objet de contrôles réglementaires. Cette étude nous a permis d'éliminer les méthodes qui ne correspondaient pas à nos demandes. Les méthodes retenues ont été testées afin d'évaluer leur efficacité.

Cet article présente donc, dans un premier temps, d'une manière synthétique les méthodes de décontamination proposées dans la littérature avec leurs applications, leurs avantages, leurs inconvénients et leur faisabilité. La deuxième partie présente les premiers essais de qualification des méthodes retenues, les résultats et les conclusions que l'on peut en tirer. Enfin la dernière partie de cet article fait le bilan des recherches et des essais effectués afin de pouvoir évaluer 
les éventuels travaux à mettre en œuvre pour arriver au but de ce projet, à savoir la décontamination systématique des colis de déchets radioactifs par voie sèche.

\section{2. Étude préliminaire des procédés de décontamination existants}

Différentes études sur le nettoyage ou la décontamination de solide, dans le domaine de la radioactivité ou non, sont décrites dans la littérature. Ce présent rapport, aborde tout d'abord la décontamination par laser, puis par ultrasons (avec et sans bain), par liquides supercritiques et enfin par champs électrostatiques pulsés.

\subsection{Laser}

Il existe différents types de laser : les lasers à cristaux comme le laser YAG, les lasers à gaz comme les excimers, les lasers à électron libre (LEL), et les lasers à fibres. Dans le cas de la décontamination nucléaire, le laser le plus utilisé est celui à fibres. Zapka et al. (1991) ont décrit le mécanisme de cette décontamination. Sous les radiations laser, les particules vibrent ce qui diminue l'adhésion de la particule sur la surface. Parallèlement la surface vibre induisant le transfert momentané de la particule. Sous atmosphère humide, il existe un autre mécanisme important. L'humidité ambiante peut créer un film liquide entre la particule et la surface. Sous l'effet de la radiation laser, ce film «chauffe » et à tendance à s'expendre et exploser. Le choc créé par l'explosion cause l'éjection de la particule (Zapka et al., 1993) (Fig. 1).

Les lasers excimer produisent des impulsions courtes avec un haut flux de radiations. Ils se composent d'un laser $\mathrm{XeCl}$ ou $\mathrm{KrF}$, un réseau de fibres, un système optique, un système d'aspiration avec un filtre pour récolter les particules et un contrôle informatique. En cas des contaminations radioactives, les sources laser doivent être placées à quelques mètres de surface, il est alors nécessaire d'utiliser un système pour focaliser le rayon. Le faisceau laser est connecté au système d'aspiration, le tout est installé sur un robot pour permettre au système d'être mobile (1 m/s) (Delaporte et al., 2003). De nombreuses fibres sont testées sous différents paramètres pour déterminer les meilleures conditions.

Delaporte et al. (2003) ont testé ce laser pour décontaminer différentes surfaces. Ils ont utilisés trois types d'échantillons pour tester le laser :

- acier inoxydable et inconel soumis à de la vapeur générée par un réacteur nucléaire, une fine couche d'oxyde est crée à haute température $\left(300{ }^{\circ} \mathrm{C}\right) \mathrm{de}$ l'eau et haute pression (170 bar). Les principaux radionucléides trouvés sont le cobalt-60, le nickel-60 et le fer-60 ; 


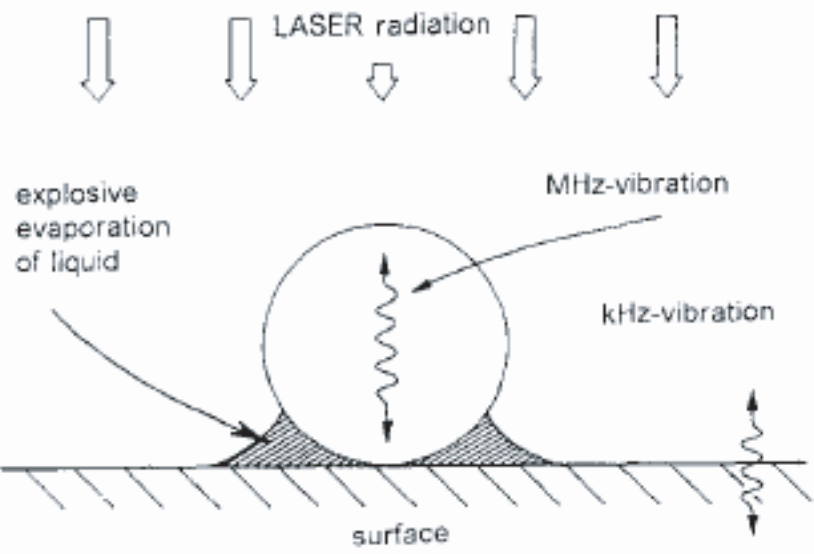

Figure 1 - Mécanismes de nettoyage au laser (Zapka et al., 1991).

Mechanisms of laser cleaning (Zapka et al., 1991)

- ils ont également irradiés des échantillons d'aluminium, dans ce dernier cas la couche d'oxyde est formée à température ambiante et à pression atmosphérique. Les principaux radionucléides piégés dans la couche sont le antimoine-125, l'europium-154 et le césium-137.

L'enlèvement de la couche d'oxyde est nécessaire afin de décontaminer complètement.

Plusieurs séries d'impulsions laser ont été testées à différentes fluences (énergie par $\mathrm{cm}^{2}$ ) afin de déterminer le couple le plus efficace. Il apparaît que les meilleurs taux de décontamination (entre 80 et $97 \%$ ) sont obtenus avec une fluence de $2,5 \mathrm{~J} / \mathrm{cm}^{2}$ après 1000 impulsions lasers. Ils ont remarqué que le pourcentage de décontamination dépend de la nature de l'échantillon, de l'élément et $\mathrm{du}$ processus de contamination. Cette technique présente un avantage important : aucune production d'effluent, seul le filtre du système d'aspiration constitue un déchet. De plus, ce laser ne crée pas de dégâts à la surface ce qui est important puisque les colis sont ensuite transportés et entreposés. Ces colis ne doivent donc pas être endommagés.

\subsection{Les ultrasons}

Il en existe 2 types : les ultrasons (utilisés dans des applications classiques) et les ultrasons à « secs ». Les premiers utilisent un bain rempli de liquide (généralement de l'eau) et les seconds n'ont pas besoin de liquide et sont appelés ultrasons « à $\sec »$. 


\section{FLUIDE}

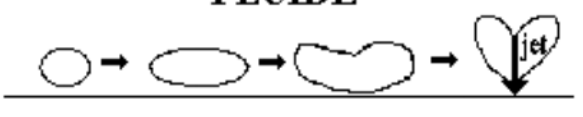

SOLIDE
Figure 2 - Évolution de la cavité d'une bulle à proximité d'une surface solide. Evolution cavitations of a bubble near a solid surface.

\subsubsection{Ultrasons en phase liquide}

La décontamination ou le nettoyage par ultrasons est basé sur le principe de la cavitation.

La cavitation acoustique décrit l'apparition d'une population de bulles de gaz sous l'influence d'une onde acoustique. Quand un liquide est soumis à des ondes ultrasonores suffisamment intenses, apparaissent des bulles gazeuses de très petites dimensions, provoquées par une pression locale inférieure à la pression ambiante. Quand la pression redevient positive, ces bulles implosent en moins d'une microseconde, induisant localement une température de plusieurs milliers de degrés et une onde de pression de plusieurs milliers de fois la pression atmosphérique. Lorsque les bulles de cavitation rencontrent une surface solide, elles implosent sur cette surface en formant des micro-jets de liquide très violents $(100 \mathrm{~m} / \mathrm{s}$ ) qui décapent la surface solide (SINAPTEC, 2008) (Fig. 2).

L'énergie développée par la cavitation ultrasonore combine plusieurs effets produisant :

- le développement de forces près de la surface à nettoyer qui induisent l'apparition de violents micro-jets liquides orientés vers la surface à nettoyer,

- le nettoyage dans les pores et crevasses de la surface,

- la dispersion des contaminants dans le fluide sous l'influence de mouvements turbulents provoqués par le champ ultrasonore.

Des recherches sur la décontamination de sols et des sédiments par des ultrasons (Collings et al., 2006) montrent qu'après 60 minutes sous ultrasons $85 \%$ de PCB et $70 \%$ des composés organiques ont été enlevés. Ils ont remarqué que l'orientation était un paramètre important puisque si les solides contaminés passent dans la zone de cavitations, les taux de décontamination sont alors proches de $90 \%$. Un grand nombre de polluants : atrazine, simazine, hydrocarbures... ont été enlevés avec cette méthode.

Le nettoyage par ultrasons semble efficace, particulièrement s'il est couplé avec une autre méthode ou avec des produits chimiques (Zhao et al., 2007 ; Farmer et al., 2000 ; Chai et al., 1999). Les taux de décontamination dépendent de nombreux facteurs comme la fréquence, la puissance... 


\subsubsection{Ultrasons à sec}

Le principe est celui de l'usinage ultrasonique. Une étude (Thoe et al., 1998) montre que ce processus est non thermique, et qu'il n'est pas à relier à la conductivité de la surface à traiter. Les matériaux ne sont pas altérés chimiquement. C'est donc un processus qui n'entraîne pas de dommage sur la pièce. Le processus commence par la conversion d'énergie électrique à basse fréquences en un signal électrique de haute fréquence, qui est ensuite introduit dans un transducteur. Ce transducteur convertit l'énergie électrique haute fréquence en vibrations mécaniques. Cela entraine la vibration de l'outil le long de l'axe longitudinal, à haute fréquence (supérieure ou égale à $20 \mathrm{kHz}$ ) avec une amplitude de 5 à $50 \mu \mathrm{m}$. La gamme typique de puissance utilisée est $50-3000 \mathrm{~W}$ et peut atteindre $4 \mathrm{~kW}$ dans quelques appareils.

Ils sont notamment utilisés pour le nettoyage de l'air (Mason, 2007 ; Gallego et al., 1994). Gallego et al. (1994) ont développé un nouveau type des générateurs de sons puissants où les ultrasons atteignent une énergie de transmission beaucoup plus efficace. Il se compose d'une plaque vibrante circulaire et d'un vibreur piézoélectrique activé au centre. Ce type d'appareil a été utilisé pour la précipitation des poudres en suspension dans l'air. Des tests (Gallego et RieraFranco De Sarabia, 1999) sur des particules de fumée de charbon ont donné une efficacité de collecte en masse de $93 \%$ pour une consommation d'énergie de $3 \mathrm{kWh} / 1000 \mathrm{~m}^{3}$.

Les ultrasons à secs peuvent être utilisés pour décontaminer l'air mais aussi des surfaces solides (Thoe et al., 1998). Dans tous les cas, une énergie électrique est convertie en vibrations mécaniques grâce à un transducteur. Cette technique est efficace et non destructive. Elle est notamment utilisée dans le dépoussiérage de conduits présents dans les centrales nucléaires. Dans ce cas, on a un peu le même effet qu'une soufflerie: les ultrasons font vibrer la surface ce qui éjecte les particules. En adaptant certains paramètres, comme la fréquence ou la puissance, on pourrait transposer cette méthode à notre application.

\subsection{Fluides supercritiques}

Quand un fluide est dans des conditions de température et de pression supérieure à son point critique, il entre dans un état appelé « supercritique ». Les changements d'état gaz/liquide et liquide/fluide supercritique se font de manière continue. Ces fluides ont des propriétés différentes de celles d'un gaz ou d'un liquide mais qui sont comprises entre les deux. Ils ont une viscosité proche de celle d'un gaz, une densité proche de celle d'un liquide avec un pouvoir de diffusivité très élevé par rapport au fluide liquide ce qui facilite leur pénétration dans des milieux poreux. 
Un fluide supercritique présente un autre avantage par rapport aux autres solvants : sa solubilité change selon que l'on fait varier sa température ou sa pression. On peut ainsi faire en sorte qu'il soit un solvant pour certaines substances à un moment donné, et plus du tout l'instant d'après. Cela facilite la récupération de la substance qui a été dissoute (Samvura et Totté, 2007).

Ces caractéristiques permettent d'adapter le fluide au matériau que l'on souhaite nettoyer. Dans ce procédé, on peut utiliser des fluides de différentes natures : dioxyde de carbone, hexafluorure de soufre, oxyde nitreux, alcanes légers (de un à cinq atomes de carbones)... Cependant, le dioxyde de carbone est le plus utilisé car il présente l'avantage d'une mise en œuvre relativement facile : il est bon marché, non toxique, ininflammable et possède des conditions critiques facilement accessibles (pression critique : 7,3 Mpa et température critique : 31,1 ${ }^{\circ} \mathrm{C}$ (Meynier et Malherbe, 2003).

L'utilisation des fluides supercritiques est d'un grand intérêt dans les domaines des applications industrielles, de la pharmacie, de l'agroalimentaire, de l'élaboration de matériaux et de l'environnement. La diversification des applications offertes par l'utilisation des fluides supercritiques repose principalement sur leurs propriétés spécifiques concernant les phénomènes de solvatation sélective, de solubilité ou de réactivité. Les premières applications des fluides supercritiques concernaient tout naturellement le remplacement des solvants organiques dans les procédés d'extraction. Cette technique permet de travailler à une température modérée, ce qui ne dénature pas l'extrait obtenu, il reste dans un état proche du naturel. Elle permet d'autre part d'obtenir des extraits exempts de tous résidus de solvant d'extraction. À la fin de l'extraction, par abaissement de la pression, on provoque le passage du gaz carbonique de l'état supercritique à l'état gazeux et le $\mathrm{CO}_{2}$ s'élimine tout seul de l'extrait sous pression atmosphérique.

Cette méthode présente de nombreux avantages :

- diminution des déchets secondaires par la non-utilisation de solvants organiques,

- les complexes de métaux extraits sont rapidement et complètement séparés du milieu par gazéification du $\mathrm{CO}_{2}$,

Cependant des études, dans le domaine du nucléaires, sur la décontamination de déchets solides par un fluide supercritique (Meguro et al., 2004 ; Shamsipur et al., 2001 ; Tomioka et al., 2000) ont montrées que, dans la plupart des cas, pour augmenter l'efficacité de cette technique, il fallait ajouter des agents de chélation phosphate de tri-n-butyle (TBP), oxyde de trioctyl phosphine (TOPO)... qui doivent être stables, avec une cinétique rapide, une forte solubilité... Shamsipur et al. (2001) 
augmente la solubilité avec l'augmentation du pouvoir de solvatation par addition de différents solvants organiques polaires appelés modificateurs. L'ajout de ces modificateurs et de ses agents de chélation n'est pas en adéquation avec nos critères puisqu'il y a utilisation de solvants organiques qui devront être retraités. De plus les travaux bibliographiques ne donnent pas des résultats assez satisfaisants pour notre étude : sans co-extracteur extraction de seulement $12 \%$ de l'uranium, avec du TBP comme modificateur et de l'acide di(2-ethylhexyl) phosphorique (HDEPH) comme agent chélateur : extraction de moins de $65 \%$ (Shamsipur et al., 2001). Cette technique n'est donc pas utilisable dans le cadre de notre étude.

\subsection{Champs électrostatiques à impulsions}

Ce procédé utilise un précipitateur électrostatique qui fait appel à un champ électrique. Les particules, en suspension dans un gaz, qui passent entre des électrodes de charge opposée, sont chargées électriquement et ensuite captées pour être séparées de ce gaz (ASHRAE, 1992).

L'énergie électrique est nécessaire pour l'ionisation du gaz, le chargement des particules, la coagulation des particules ou l'agglomération, ou la condensation de la vapeur. La décharge électrique de ce type d'électrodes, habituellement maintenue à un haut potentiel négatif, produit des ions gazeux par attachement des électrons libres aux molécules de gaz. C'est la collision des particules de gaz avec les particules qui les charge. Il existe deux mécanismes importants pour le chargement des particules par courant ionique :

- le champ de charge par lequel les ions sont conduits aux particules en raison d'une force électrostatique crée par un champ électrique externe,

- la diffusion de charge qui est due à l'énergie cinétique des ions gazeux qui bombardent les particules indépendamment du champ électrique.

Pour les particules de plus de $1 \mu \mathrm{m}$, le champ de charge est le mécanisme prépondérant alors que pour celles de moins de $0,1 \mu \mathrm{m}$ la charge est principalement due à la diffusion des ions. Mais une étude (Jaworek et al., 2007) a montré la difficulté de recueillir des particules de l'ordre de 0,1-1 m. L'efficacité de la collecte est inférieure à $90 \%$ pour ces particules. Ainsi, un ESP avec deux étapes a été mis au point où le chargement et la précipitation sont deux processus séparés. Des ESP (Précipateur ElectroStatique) à trois ou quatre étapes pour la collecte des cendres volantes ont été testées (Jaworek et al., 2007), l'efficacité du nombre de particules collectées est supérieure à $96 \%$, tandis que l'efficacité de collecte en masse a été plus élevée : 99,99 \%. La faible cohésion de la poussière, en raison du manque de courant de décharge est principalement responsable du réentraînement des particules de l'électrode de collecte. Ainsi, des agents de surface ont été proposés pour éviter ce réentraînement. Kawaka et al. (1998) utilisent de l'eau 
avec un surfactant non ionique ce qui réduit la tension superficielle. Cet agent est pulvérisé en amont et améliore l'adhérence entre les particules et l'électrode, en particulier pour les grandes particules.

Une autre façon de lutter contre les particules fines est d'accroitre leur taille moyenne avant les précipitations. L'agglomération est un processus dans lequel les petites particules se rassemblent (coagulation) pour en former une plus grande ou en petites particules qui adhèrent les unes aux autres. Dans un premier temps, les particules sont chargées de polarité opposée, et dans un second temps, elles sont soumises à un champ électrique alternatif, ce qui entraîne la collision des particules. Un champ électrique alternatif favorise l'agglomération des particules chargées en plus de celles crées par le mouvement brownien. Certaines études montrent que la tension a une importance sur l'efficacité moyenne de collecte (St Georges et Feddes, 1995 ; Tanaka et Zhang, 1995). Ils affichent des taux de collecte d'environ $90 \%$ dans des conditions optimales. Cette méthode n'a pas encore été étudiée et testée dans l'industrie nucléaire. En adaptant certains paramètres (puissance, couplage avec une autre méthode...) on pourrait l'appliquer à la décontamination de surfaces solides. Le colis pourrait alors être relié à l'une des bornes et former ainsi une des électrodes.

\section{Bilan bibliographique}

À la suite de l'étude bibliographique, nous pouvons éliminer les fluides supercritiques et les ultrasons (ceux utilisés dans des applications classiques) car ces méthodes ne respectent pas nos exigences. En effet, les ultrasons produisent des déchets liquides et les fluides supercritiques ne sont pas suffisamment efficaces et rapides. Le recours à des ultrasons classiques entraîne la production de déchets liquides qui nécessitent un traitement important, plus le colis est important plus la quantité de liquides est importante puisque «l'objet» traité doit être complètement immergé dans le liquide. Ainsi, cette méthode ne peut pas être utilisée pour le nettoyage des colis.

Les fluides supercritiques, bien qu'ils soient non toxiques et facilement exploitables avec un coût de revient acceptable, n'ont pas une efficacité suffisante sauf si l'on ajoute un tensioactif ou autres produits chimiques, qui constituent des déchets secondaires, qui doivent ensuite être traités. Ainsi, nous pouvons éliminer la décontamination par fluides supercritiques et par ultrasons classiques.

Les autres méthodes : les lasers, les ultrasons à sec ou avec un taux d'humidité relativement maîtrisé et les champs électrostatiques pourraient répondre à nos exigences. Ces méthodes sont efficaces, rapides, sans production d'effluents liquides et tout à fait maîtrisable pour l'intégrité de l'enveloppe des colis des déchets. L'utilisation des champs électriques pour le nettoyage semble simple, 
rapide et efficace. Certaines modifications du processus peuvent être faites en vue de leur application pour la décontamination des colis notamment la puissance, et la fréquence du champ. Les ultrasons à sec présentent un avantage important par rapport aux ultrasons en phase liquide puisque cette méthode ne nécessite pas de liquide. Elle est basée sur le principe des vibrations acoustiques qui éjectent les particules. Dans cette méthode, quelques modifications seront également nécessaires pour l'appliquer au cas de la décontamination des colis : taille de la sonotrode, fréquence, puissance... Enfin, dans la littérature, la décontamination laser semble la meilleure méthode car elle est rapide et très efficace. Aucun déchets n'est crée et des études montrent que la surface du colis ne présente pas de dommage. Les ultrasons et les champs électrostatiques ont cependant l'avantage d'occuper une petite surface ( $1 \mathrm{~m}^{2}$ est suffisant pour poser l'appareil) alors que le laser à fibres nécessite un peu plus et le laser excimer plusieurs mètres carrés! Du point de vue du prix, les ultrasons et les champs électrostatiques sont plus abordables que le laser : environ $11 \mathrm{k} €$, le double pour les lasers à fibres et environ dix fois plus pour les lasers excimers. Les ultrasons et les champs électrostatiques présentent également l'avantage d'être plus facilement manipulables que les lasers excimers. De cette étude bibliographique, il en ressort donc que la méthode de décontamination par laser est celle qui semble la plus efficace mais les ultrasons et les champs électrostatiques sont plus faciles à utiliser, il apparaît donc raisonnable de tester ces trois méthodes pour déterminer la méthode la plus adaptée à notre problématique. En raison de la complexité d'utilisation des lasers excimers, aucun essai n'a été effectué avec ce type de lasers, seul le laser à fibres a été testé.

\section{Essais de qualification}

Afin de déterminer la méthode la mieux adaptée à notre problématique, nous avons effectué des essais préliminaire afin d'évaluer leur faisabilité ainsi que leur efficacité. Des essais préliminaires ont donc été effectués, dans différents laboratoires et entreprises, sur les lasers à fibres, les ultrasons à sec et les champs électrostatiques. Pour ces essais, deux matériaux et deux éléments ont été choisis : l'aluminium et l'inox et le cobalt et le césium. Chaque méthode de décontamination a suivi le même protocole expérimental décrit ci-dessous. Il faut noter que ces laboratoires nous ont accueillis pour que l'on puisse évaluer leur appareil par rapport à notre problématique. Aucune coopération ou sous-traitance n'a été mise en place, c'est pourquoi chaque type d'essai ne compte qu'un seul échantillon et un témoin par matière. On nomme échantillons tests, les échantillons ayant subit tout le protocole : contamination / décontamination / récupération. Les échantillons "témoins », eux n'ont pas été décontaminés par les techniques étudiées, ils ont seulement subit la contamination et la récupération. 


\subsection{Matériel et méthode}

Les radioéléments et la nature des surfaces ont été choisis en fonction des possibilités du laboratoire et de la représentativité de ces échantillons avec la réalité. Ainsi le cobalt composant plus de $90 \%$ de la contamination présente sur les colis, il nous a semblé judicieux de l'étudier. Bien que la proportion de Cs dans la contamination soit beaucoup moins importante, nous l'avons également étudié pour pouvoir comparer le comportement de deux éléments sur un même matériau. Du point de vue de la nature des surfaces, les colis sont principalement composés d'acier et de béton, cependant en raison de la complexité du béton (surface poreuse...), seul l'acier a été gardé comme matériau « représentatif ». Enfin, afin d'avoir un second point de comparaison pour étudier les différences de comportement d'un même élément sur deux matériaux, nous avons également étudié l'aluminium.

Il existe actuellement deux voies de contamination des colis : la première est la voie humide et la seconde par aérosol. L'étude de ces deux voies est importante cependant, nous avons d'abord préféré étudier la contamination par voie humide. La présente étude ne fait donc été que des essais de décontamination suite à une contamination humide.

Les échantillons seront répartis en deux groupes : un premier composé des témoins (aluT et inoT), le second sera composé des «véritables » échantillons (aluE et inoE) ayant subit la décontamination.

La solution contaminante est une solution aqueuse comprenant du cobalt et du césium. Elle a été préparée à partir de :

- chlorure de cobalt hexahydraté,

- nitrate de césium.

Un litre de solution a été préparée, les éléments ayant une concentration de $10^{-4} \mathrm{M}$.

Ces choix ont été définis en fonction des stocks du laboratoire au moment de l'étude.

- Phase de contamination. Les plaques sont disposées séparément dans des boîtes de pétri remplie de la solution contaminante. Elles sont ainsi laissées pendant 72 heures (Fig. 3). Ensuite les échantillons sont mis à sécher jusqu'à utilisation. La solution restante (SR) de chaque boîte de pétrie est recueillie dans un flacon afin d'être analysée (Fig. 4).

- Les échantillons tests sont ensuite décontaminés selon la méthode choisie : laser, ultrasons ou champs électrostatiques. 


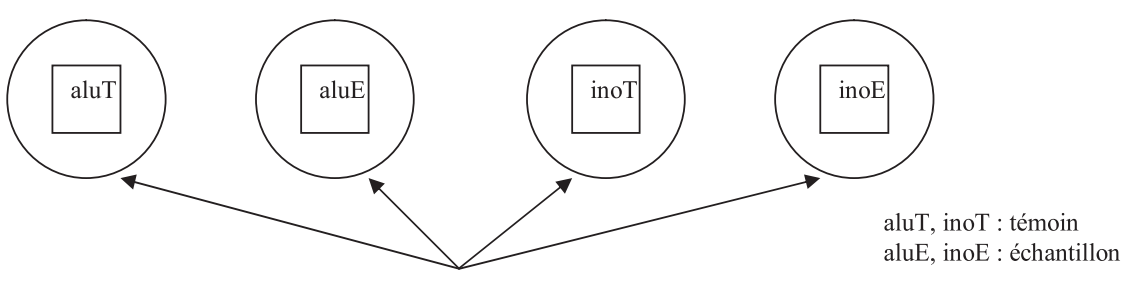

Boîtes de pétri remplies de solution de Co et Cs

Figure 3 - Étapes de la contamination.

Contamination step.

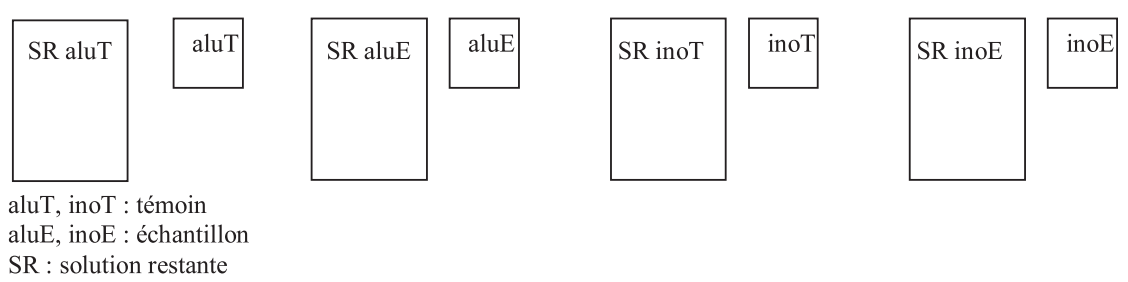

Figure 4 - Récupération de la solution restante.

Recovery of the remaining solution.

- Phase de récupération. Les échantillons et les témoins sont immergés dans une solution aqueuse d'acide nitrique à $2 \%$ ultra pure pendant $24 \mathrm{~h}$. Ensuite la solution est récupérée et filtrée à l'aide de filtre seringue. Les solutions ainsi récupérées (STaluT, STaluE, StinoT, STinoE) sont ensuite diluées afin d'être injectées en ICP-MS. Il en est de même des solutions restantes après contamination (SRaluT, SRaluE, SRinoT et SRinoE) (Fig. 5).

\subsection{Résultats}

\subsubsection{Méthode de calculs}

L'efficacité de chaque méthode a été déterminée en calculant les taux de décontamination. Ceux-ci sont calculés à partir des témoins et des échantillons. En effet, l'absorption des éléments sur les matériaux étant très faible (de l'ordre de $5 \%$ ), il est judicieux de calculer ce taux de décontamination en comparant la concentration d'éléments présents sur la plaque «témoin», c'est-à-dire non décontaminée et celle restant sur les échantillons tests. 

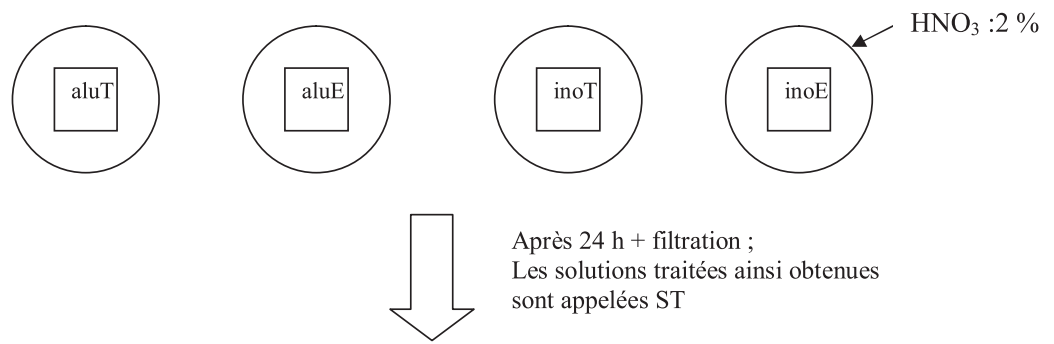

Après $24 \mathrm{~h}+$ filtration ;

Les solutions traitées ainsi obtenues

sont appelées ST
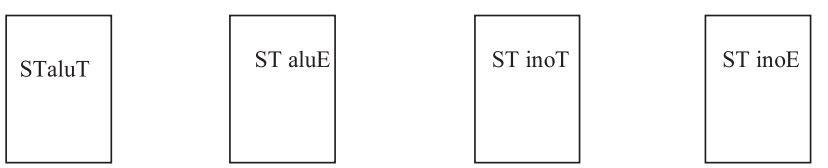

Dilution

puis
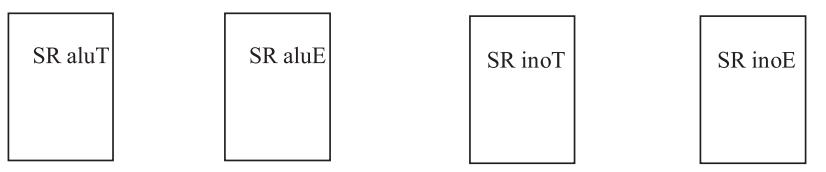

injection ICP-MS

Figure 5 - Étapes de récupération.

Recovery step.

Ainsi, la détermination des taux de décontamination se fait selon les calculs suivants :

- calcul du pourcentage de contamination résiduelle :

$\%$ de contamination résiduelle en $\mathrm{Co}=100 \times \frac{\mathrm{ppb} \text { de Co échantillon }}{\mathrm{ppb} \text { de Co témoin }}$,

$\%$ de contamination résiduelle en $\mathrm{Cs}=100 \times \frac{\mathrm{ppb} \text { de Cs échantillon }}{\mathrm{ppb} \text { de Cs témoin }}$;

- calcul du pourcentage de décontamination :

$\%$ de décontamination $\mathrm{Co}=100-\%$ de contamination résiduelle de Co,

$\%$ de décontamination $\mathrm{Cs}=100-\%$ de contamination résiduelle de Cs.

\subsubsection{Bilan des résultats}

Les essais de décontamination laser ont été effectués avec un laser «LASERBLAST » de la société Quantel située à Paris. Ceux par ultrasons par un 

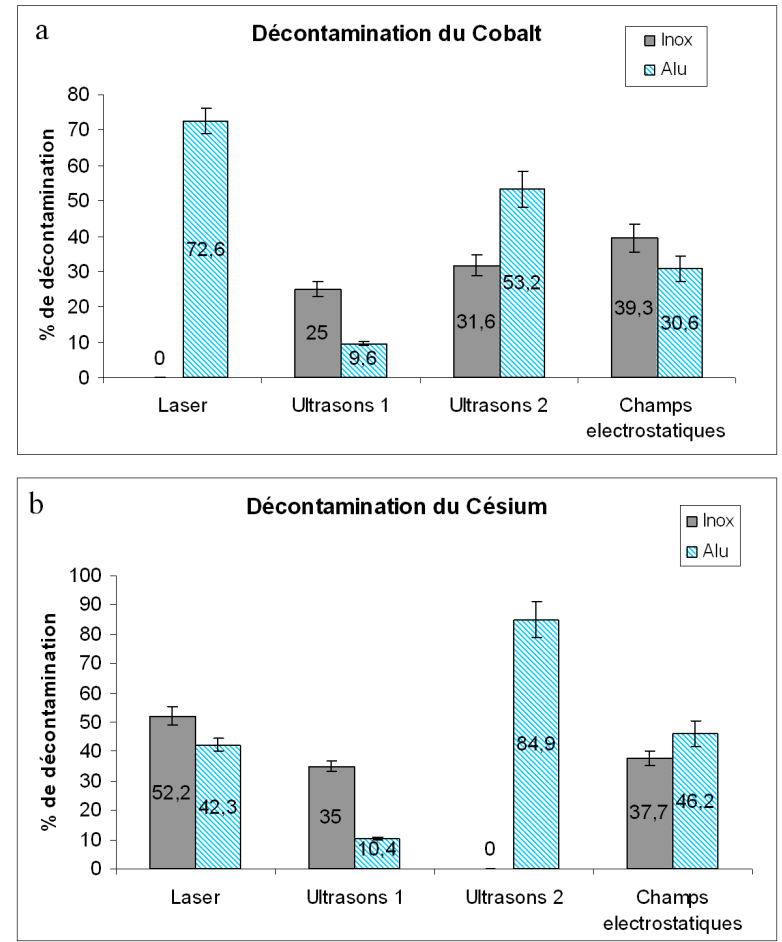

Figure 6 - Pourcentages de la décontamination de (a) Co et (b) Cs.

Percentages of decontamination of (a) Co and (b) Cs.

appareil de la société SINAPTEC à Lilles et la décontamination par champs électrostatiques a été effectuée à l'IUT d'Angoulême au département Qualité logistique industrielle et organisation (QLIO). Dans le cas de la décontamination par ultrasons, deux séries de tests ont été effectuées. La première totalement à « sec » (nommée essais ultrasons 1 par la suite) et la seconde avec vaporisation d'eau (nommé essais ultrasons 2 par la suite) avant exposition aux ultrasons. Pour les champs électrostatiques, différentes fréquences et deux distances entre les électrodes ont été testés afin d'estimer les paramètres optimum.

Pour le cobalt, les méthodes les plus efficaces semblent être le laser et les ultrasons avec vaporisation. On peut faire les mêmes observations pour la décontamination du césium. Dans tous les cas la décontamination par ultrasons à sec sans vaporisation et les champs électrostatiques donnent les résultats les moins bons (Fig. 6). 
Ces essais préliminaires nous ont permis de tirer plusieurs conclusions. Tout d'abord, l'efficacité de la décontamination dépend de plusieurs paramètres notamment la nature de l'élément mais aussi celle du substrat. En effet un élément va se comporter différemment sur des matériaux différents et deux éléments se comporteront différemment sur un même matériau. Ceci montre l'importance des propriétés physico-chimiques des éléments et des phénomènes d'absorption/désorption.

\section{Conclusions et perspectives}

L'étude bibliographique et les essais préliminaires pour la recherche d'une méthode de décontamination des colis avant transport a permis d'évaluer la faisabilité et l'efficacité des méthodes étudiées. Dans la littérature, les meilleurs résultats sont obtenus avec les lasers. Pour les essais préliminaires, les meilleurs résultats sont obtenus avec les lasers et les ultrasons à sec avec vaporisation. $\mathrm{Au}$ niveau de la facilité de mise en œuvre, du cout et de l'encombrement, il semble que les ultrasons à sec soient la méthode la plus adaptés à notre problématique. Afin de valider cette hypothèse, des essais plus poussés seront effectués.

Par ailleurs ces recherches ont montré l'importance de la nature des contaminants, du matériau support, des mécanismes d'adhésion et du détachement des particules sur la surface. Tout d'abord, le dépôt de particules sur la surface est un paramètre important. Les mécanismes d'absorption des contaminants sur la surfaces influencent le processus de décontamination, il serait alors judicieux de faire des simulations numériques sur l'écoulement des fluides en mode statique et dynamique. Le type de surface joue également un rôle important. La surface peut être poreuse, dans ces cas, les particules peuvent y entrer et sont alors plus difficiles à enlever. Tous ces paramètres influencent la décontamination, il est donc important de les connaître pour prévoir et améliorer les protocoles industriels. Une étude approfondie sur les mécanismes d'adhésions des particules devra donc être menée.

Cette étude pourrait ainsi aboutir à une méthode innovante de décontamination surfacique des colis de déchets radioactifs afin d'assurer et de garantir la non contamination surfacique jusqu'à leur destination : centre de traitement et/ou stockage. La solution retenue devra être efficace, rapide, non destructive et sans production d'effluent liquide. L'activité labile surfacique des colis devra être piégée sur un filtre absolu sans aucune dissémination.

\section{REFERENCES}

ASHRAE (1992) Systems and Equipment Handbook, SI Edition, Atlanta.

Chai X., Kobayashi T., Fujii N. (1999) Ultrasound-associated cleaning of polymeric membranes for water treatment, Separation and Purification Technology 15, 139-146. 
Collings A.F., Farmer A.D., Gwan P.B., Pintos A.P.S., Leo C.J. (2006) Processing contaminated soils and sediments by high power ultrasound, Min. Engineer. 19, 450-453.

Delaporte P., Gastaud M., Marine W., Sentis M., Uteza O. (2003) Dry excimer laser cleaning applied to nuclear decontamination, Appl. Surf. Sci. 208-209, 298-305.

Farmer A.D., Collings A.F., Jameson G.J. (2000) Effect of ultrasound on surface cleaning of silica particles, Int. J. Min. Proc. 60, 101-113.

Gallego J.A., Rodriguez G., San Emeterio J.L., Montoya F. (1994) Electroacoustic unit for generating high sonic and ultrasonic intensities in gases and interphases, USA Patent No. 5299175.

Gallego J.A., Riera-Franco De Sarabia E.M. (1999) Application of Acoustic Agglomeration to Reduce Fine Particle Emissions from Coal Combustion Plants, Environ. Sci. Technol. 33, 3843-3849.

Jaworek A., Krupa A., Czech T. (2007) Modern electrostatic devices and methods for exhaust gas cleaning: A brief review, J. Electrost. 65, 133-155.

Kawada Y., Jindai W., Zukeran A., Ehara Y. (1998) Influence of Lypophile on preventing reentrainement in spraying surfactant type electrostatic precipitators, Seventh International Conference on Electrostatic Precipitation, 20-25 septembre 1998, pp. 76, 77, 83.

Mason T.J. (2007) Developments in ultrasound - Non-medical, Progr. Biophys. Mol. Biol. 93, 166 175.

Meguro Y., Iso S., Yoshida Z., Tomioka O., Enokida Y., Yamamoto I. (2004) Decontamination of uranium oxides from solid wastes by supercritical $\mathrm{CO}_{2}$ fluid leaching method using $\mathrm{HNO}_{3}-\mathrm{TBP}$ complex as a reactant, J. Supercrit. Fluids 31, 141-147.

Meynier B., Malherbe J. (2003) Synthèse bibliographique : Les bois traités. M.S.T. « Mesures et Traitements des Pollutions et Nuisances, Thèse de l'université de Pau et des pays de l'Adour.

Samvura J.C., Totté A. (2007) Utilisation du $\mathrm{CO}_{2}$ supercritique dans le domaine de l'agroalimentaire, Pôle Technologique Agro-Alimentaire, Newsletter $n^{\circ} 10$.

Shamsipur M., Ghiasvand A.R., Yamini Y. (2001) Extraction of uranium from solid matrices using modified supercritical fluid $\mathrm{CO}_{2}$, J. Supercrit. Fluids 20, 163-169.

SINAPTEC (2008) Nettoyage Sous Ultrasouns, http://www.sinaptec.fr/production/nettoyage.htm.

St Georges S.D., Feddes J.J.R. (1995) Removal of airborne swine dust by electrostatic precipitation, Can. Agricult. Eng. 37, 103, 104, 107.

Tanaka A., Zhang Y. (1995) Efficiency of a negative ionization system on dust settling and the electrostatic effect in a confinement swine building, ASAE Paper $\mathrm{N}^{\circ} 954481$.

Thoe T.B., Aspinwall D.K., Wise M.L.H. (1998) Review on ultrasonic machining, Int. J. Mach. Tools Manufact. 38, 239-255.

Tomioka O., Enokida Y., Yamamoto I., Takahashi T. (2000) Cleaning of materials contaminated with metal oxides through supercritical fluid extraction with $\mathrm{CO}_{2}$ containing TBP, Progr. Nucl. Energy 37, 417-422.

Zapka W., Tam A.C., Ziemlich W. (1991) Laser cleaning of wafer surfaces and lithography masks, Microelectr. Engineer. 13, 547-550.

Zapka W., Ziemlich W., Leung W.P., Tam A.C. (1993) "Laser cleaning" removes particles from surfaces, Microelectr. Engineer. 20, 171-183.

Zhao H.L., Wang D.X., Cai Y.X., Zhang F.C. (2007) Removal of iron from silica sand by surface cleaning using power ultrasound, Min. Engineer. 20, 816-818. 